\title{
Approximate solution methods for spectral radiative transfer in high refractive index layers
}

\author{
R. SIEGEL† and C. M. SPUCKLER + \\ $\dagger$ Research Academy and $\ddagger$ Internal Fluid Mechanics Division, NASA Lewis Research Center. \\ Cleveland, OH 44I35. U.S.A.
}

\begin{abstract}
Some ceramic materials for high temperature applications are partially transparent for radiative Iransfer. The refractive indices of these materials can be substantially greater than one which influences internal radiative emission and reflections. Heat transfer behavior of single and laminated layers has been obtained in the literature by numerical solutions of the radiative transfer equations coupled with heat conduction and heating al the boundaries hy convection and radiation. Two-flux and diffusion methods are investigated here to obtain approximate solutions using a simpler formulation than required for exact numerical solutions. Isotropic scattering is included. The two-flux method for a single layer yields excellent results for gray and two band spectral calculations. The diffusion method yields a good approximation for spectral behavior in laminated multiple layers if the overall optical thickness is larger than about ten. A hybrid spectral model is developed using the two-flux method in the optically thin bands, and radiative diffusion in bands that are optically thick.
\end{abstract}

\section{INTRODUCTION}

SOME of the ceramic materials being developed and evaluated for high temperature applications are partially transparent to radiant energy. For high $\mathrm{tcm}$ perature conditions such as in a combustion chamber. infrared anc visible radiation from the surroundings penetrates into the material heating it internally. Since temperatures in the material are elevated, internal radiant emission becomes significant; this is especially true for materials with high refractive indices since internal emission depends on the refractive index squared. In addition to emission, energy transfer within the material depends on internal radiant absorption and scattering. and on heat conduction. As a result, radiant energy can affect internal temperatures of some ceramic engine parts and coatings that partially transmit radiation in portions of the wavelength spectrum. It must be determined when radiative processes can be importint. and how large an effect they have when compared with calculations when materials are assumed opaque.

The refractive indices of single or composite materials can have a considerable effect. Surface reflections depend on the ratio of refractive indices across an interface; this affects the amount of external radiation transmitted into a material, and the amount reflected from internal interfaces in a composite. Since emission in a material depends on its refractive index squared, internal emission can be many times that from a blackbody radiating into a vacuum. So that radiation leaving through an interface does not exceed that of a blackbody, there is substantial energy reflection at the internal surface of the interface. mostly by total internal reflection. Scattering is another means for energy transfer in the layer; it interacts with the internally reflected energy and alters the temperature distribution.

There is an extensive literature on radiative transfer in planc layers. Much of the work has been for gases with refractive indices of one although some of the carly work for predicting heat treating and cooling of glass plates [1] included refractive index effects. The literature has been briefly reviewed in our previous work [2-4] and is not repeated here. In refs. [2-4] temperature distributions and heat flows in partially transmitting materials are predicted by radiative analyses using the transfer equations coupled with heat conduction. The governing integral equations are solved numerically. Each exterior boundary is heated by radiation and convection, and the effect of diffuse interface reflections is included. Results were obtained for a single layer that is either gray or has a two- or three-band spectral variation of the absorption cocfficient, and for a gray two-layer composite. This simulates a ceramic layer with reinforcement by another material. and also examines the behavior of a ceramic coating used to protect another ceramic. Various amounts of isotropic scattering are included to simulate internal reffections by a granular or reinforcing structure.

The formulation and solution of the exact spectral equations of radiative transfer including scattering is rather complicated : hence it is desirable to have more convenient approximate methods if these will yield accurate results. Two-flux. diffusion, and hybrid methods are investigated here to provide a simplified formulation for obtaining approximate solutions. The 


\section{NOMENCLATURE}

a absorption coefficient of material in layer [m ']

c) velocity of radiant propagation in vacuum [ $\left.\mathrm{m} \mathrm{s}^{-1}\right]$

C constant in blackbody spectral flux

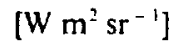

$C$ constant in blackbody spectral flux [m K] integration constant in energy equation $\left[\mathrm{W} \mathrm{m}^{-1}\right] ; C \tilde{O} N=C O N / D \sigma T_{\mathrm{g} !}^{4}$

$D \quad$ thicknesses of plane layer $[\mathrm{m}]$

$\mathfrak{e}_{\mathrm{rnh}} \quad$ blackbody spectral flux [ $\mathrm{W} \mathrm{m}^{-2}$ ]

FS blackbody fraction in band at small frequencies

$G \quad$ flux quantity defined in equation (la) $\left[\mathrm{W} \mathrm{m} \mathrm{m}^{-2}\right] ; \tilde{G}=G / \sigma T_{\mathrm{k} !}^{4}$

$G S, G L$ values of $G$ in bands at small and large frequencies

$H$ dimensionless convection-radiation parameter, $h / \sigma T_{k^{\prime}}^{3}$

$h_{1}, h_{2}$ convective heat transfer coefficients at two boundaries of layer [ $\left.\mathrm{W} \mathrm{m}^{-2} \mathrm{~K}^{-1}\right]$

$k$ thermal conductivity of layer [ $\mathrm{W} \mathrm{m} \mathrm{m}^{-1} \mathrm{~K}^{-1}$ ]

$K$ extinction coefficient of layer, $a+\sigma_{s}\left[\mathrm{~m}^{-1}\right]$

$N$ conduction-radiation parameter, $k / \sigma T_{\mathrm{g} /}^{3} D$

$n \quad$ refractive index of layer

$q \quad$ heat flux [ $\mathrm{W} \mathrm{m}^{-2}$ ] $\tilde{q}=q / \sigma T_{y 1}^{4}$

$q^{+}, q^{-}$radiative heat fluxes in positive and negative $x$ directions [W $\mathrm{m}$ ']

$q_{r} \quad$ radiative heat flux in layer [ $\left.\mathrm{W}^{-2}\right]$; $\tilde{q}_{\mathrm{r}}=q_{\mathrm{r}} / \sigma T_{\mathrm{gl}}^{4}$

$q_{\mathrm{r}}^{\mathrm{o}} \quad$ externally incident radiation flux $\left[\mathrm{W} \mathrm{m} \mathrm{m}^{-2}\right] ; \tilde{q}_{\mathrm{r}}^{\mathrm{o}}=q_{\mathrm{r}}^{\mathrm{a}} / \sigma T_{\mathrm{g} 1}^{4}$

$q S, q L$ radiative flux in spectral bands with small and large frequencies

$R(n)$ function of refractive index defined in equation (22)

$T$ absolute temperature [K]
1 dimensionless temperature, $T / T_{\mathrm{g}}$

$T_{\mathrm{g}}, T_{\mathrm{z} 2}$ gas temperatures on two sides of layer $[\mathrm{K}]$

$x \quad$ coordinate in layer $[\mathrm{m}]: X=x / D$

Greek symbols

$\kappa \quad$ optical coordinate of layer $K x$

$\kappa_{D} \quad$ optical thickness, $K D$

$v \quad$ frequency of radiation [s ']

$\rho^{\mathrm{i}}, \rho^{\circ}$ reflectivity of interface for internally or externally incident radiation

$\sigma \quad$ Stefan-Boltzmann constant [W $\mathrm{m}^{2}{ }^{2} \mathrm{~K} \quad{ }^{4}$ ]

$\sigma_{s} \quad$ scattering coefficient of layer $\left[\mathrm{m}^{1}\right]$

$\Omega \quad$ scattering albedo of layer, $\sigma_{\mathrm{s}} /\left(a+\sigma_{\mathrm{s}}\right)=\sigma_{\mathrm{s}} / K$.

Subscripts

b blackbody

c value at cutoff frequency

$D$ based on the length $D$

$f, s \quad$ first and second internal interfaces of a layer, Fig. 2

g gas on either side of laminated layer

h, s higher and smaller refractive indices

$j \quad$ index indicating $j$ th layer in a composite

$m$ the $m$ th frequency band

$r$ radiative quantity

$\mathrm{S}, \mathrm{L}$ spectral bands with small and large frequencies

tot total heat flux by conduction and radiation

$v \quad$ frequency dependent quantity

$1, J$ the first and final layers in a composite of $J$ layers

1,2 quantilies at sides $x=0$ and $D$.

\section{Superscripts}

i. o quantity incident from inside or outside

,+- flux in positive or negative $x$ dircction. two-flux equations are given in refs. $[5,6]$, and the method is shown in refs. $[7,8]$ to give accurale results for gray plane layers with a refractive index of one. The method is extended here to include refractive indices larger than one, and for heating conditions such that the boundary temperatures are not specificd and are found during the solution. The two-flux method is found to be convenient for layers of moderate optical thickness; it yields excellent results for gray and two band spectral calculations in single layers with or without isotropic scattering.

The diffusion method is extended here to spectral calculations in a multilayered composite where each layer can have a different refractive index. It yields a very good approximation for spectral heat transfer characteristics in single and multiple layers if the overall optical thickness is larger than about ten.

A hybrid spectral model is developed that uses the two-flux method in optically thin spectral bands and the diffusion method in bands that are optically thick. This also provides good agreement with exact numerical solutions. When an optically thick band is present, convergence of the iterative solution method is improved compared with the two-flux method.

\section{ANALYSIS}

The wo-flux approximarion for spectral radiative transfer

A planc layer of absorbing, emitting, and isotropically scattcring material is subjected on both 


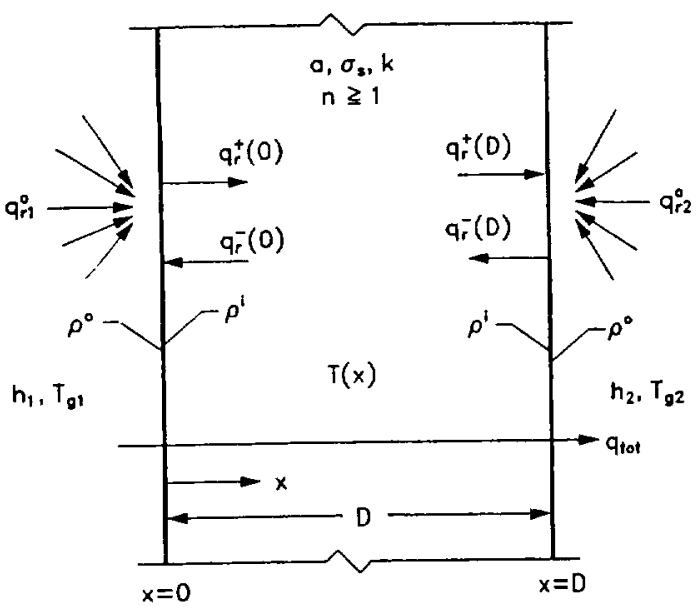

FiG. I. Geometry, coordinate system, and nomenclature for radiative fluxes in the two-flux model for heat transfer in an absorbing. emitting, and scattering semitransparent layer.

boundaries to convection and incident radiation as shown in Fig. 1. The two-flux method is used to obtain the layer temperature distribution and the heat flux transferred through the layer. The results are compared with exact numerical solutions of the radiative transfer equations to determine the accuracy of the two-flux method for a layer with a refractive index greater than one. The boundaries are assumed diffuse. The two flux equations are obtained from the radiative transfer equations as shown in refs. [5, 6 pp. 772 , 785], and are given by either the Milnc-Eddington or the Schuster-Schwarzschild approximations. These give the same functional relations, but differ by a numerical cocfficient in the radiative flux relation. The MilneEddington approximation is used here.

The two-flux equations. The two fluxes are in the positive and negative directions as shown in Fig. 1: they are each assumed isotropic and the equations are written spectrally in terms of frequency. A flux quantity $G_{r}$ and the net spectral radiative flux $q_{v r}$ are related to the two directional fluxes by the spectral relations.

$$
G_{\mathrm{v}}=2\left(q_{\mathrm{rr}}^{+}+q_{\mathrm{rr}}\right): \quad q_{\mathrm{rr}}=q_{\mathrm{rr}}^{+}-q_{\mathrm{rr}} .
$$

Equations ( 1 a) and ( $I b$ ) can be solved for the positive and negative spectral fluxes in terms of $G_{v}$ and $\psi_{\mathrm{rr}}$ : these relations will be used in the analytical development.

$$
q_{\mathrm{rr}}^{+}={ }_{2}^{1}\left(\begin{array}{c}
G_{\mathrm{v}} \\
2
\end{array}+q_{\mathrm{rr}}\right): \quad q_{\mathrm{rr}}=\frac{1}{2}\left(\frac{G_{\mathrm{r}}}{2}-q_{\mathrm{rr}}\right) .
$$

The two-flux equations including scattering are given in ref. [5] as,

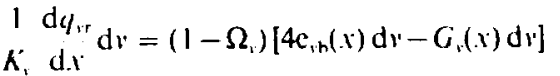

$$
\begin{aligned}
& K_{v} d d^{d} d v=-3 y / v(x) d v .
\end{aligned}
$$

The blackbody spectral flux in equation (3) contains the square of the refractive index and is given by

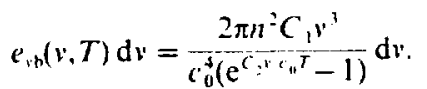

A third relation, in addition to equations (3) and (4), is the energy equation. For the present conditions of steady state without internal heat sources, the total heat flow through the layer is a constant. It is given by the sum of conduction and radiation heat flows so the energy equation is [6, p. 695],

$$
q_{101}=-k \frac{\mathrm{d} T}{\mathrm{~d} x}+\int_{r=0}^{x} q_{\mathrm{rr}} \mathrm{d} v
$$

The threc equations (3), (4) and (6). subject to proper boundary conditions, are to be solved for $q_{r}(x), G(x)$ and $T(x)$.

Equation (4) is integrated over all $v$ and the integral of $q_{i r}$ over $v$ is eliminated by using equation (6). The resulting equation is integrated over $x$ to yield

$$
q_{t+1} x=-k T(x)-\frac{1}{3} \int_{0}^{x} \frac{G_{v}(x)}{K} \mathrm{~d} v+\operatorname{CON}
$$

where $\operatorname{CON}$ is an integration constant. Evaluating equation (7) at $x=0$ and $D$ relates the boundary values of $T$ and $G$ to the values of $q_{10 t}$ and $C O N$

$$
\begin{gathered}
C O N=k T(0)+\frac{1}{3} \int_{0}^{x} \frac{G_{v}(0)}{K_{v}} \mathrm{~d} v \\
q_{1,1} D=-k T(D)-\frac{1}{3} \int_{0}^{x} \frac{G_{v}(D)}{K_{v}} \mathrm{~d} v+C O N .
\end{gathered}
$$

Equation (3) integrated with respect to $x$ will be used later in the form,

$$
\begin{aligned}
q_{r r}(x) \mathrm{d} v=q_{v r}(0) \mathrm{d} v & +K_{v}\left(1-\Omega_{v}\right) \\
& \times \int_{11}^{r}\left[4 e_{r b}(x) \mathrm{d} v-G_{v}(x) \mathrm{d} v\right] \mathrm{d} x
\end{aligned}
$$

where $e_{v \mathrm{~h}}$ is a function of $x$ as it depends on the local $T(x)$.

Boundary conditions. The boundary conditions are now developed. The total energy flow within the layer is by radiation and conduction. Because radiant absorption in the two-flux method is a volume process, there is no absorption at a boundary surface since the surface does not have any volume. Hence at each boundary the extcrnal convection is balanced only by internal heat conduction. The total energy flow by radiation and conduction within the layer is constant across the layer; the $q_{\mathrm{i} a}$ can then be expressed at the boundaries as the sum of external convection (which is equal to internal conduction) and internal radiation. This yields at $x=0$ and $D$.

$$
\varphi_{(w)}=h_{1}\left[T_{z^{1}}-T(0)\right]+\int_{0}^{1} q_{v r}(0) d v
$$




$$
q_{101}=h_{2}\left[T(D)-T_{z^{2}}\right]+\int_{0}^{x} q_{r r}(D) d v
$$

At each boundary the internal radiative flux leaving the boundary is equal to the sum of transmitted externally incident flux and reflected internally incident flux. This yields the relations at $x=0$ and $D$,

$$
\begin{gathered}
q_{\mathrm{rr}}^{+}(0)=q_{\mathrm{rr} r}^{\mathrm{o}}\left(1-\rho^{\circ}\right)+q_{\mathrm{rr}}^{-}(0) \rho^{\prime} \\
q_{\mathrm{rr}}^{-}(D)=q_{\mathrm{rr} 2}^{\mathrm{o}}\left(1-\rho^{\mathrm{a}}\right)+q_{\mathrm{rr}}^{+}(D) \rho^{\mathrm{i}} .
\end{gathered}
$$

Equations $(2 a, b)$ are used to eliminate the $q_{\mathrm{vr}}^{+}$and $q_{-;}^{-}$from equations $(12 a, b)$ to obtain expressions for $G_{v}(0)$ and $G_{v}(D)$ in terms of the radiative fluxes $q_{r r}(0)$ and $q_{\mathrm{rr}}(D)$,

$$
\begin{aligned}
G_{v}(0) & =4 \frac{1-\rho^{\mathrm{o}}}{1-\rho^{\mathrm{i}}} q_{\mathrm{rr} 1}^{\mathrm{v}}-2 \frac{1+\rho^{\mathrm{i}}}{1-\rho^{\mathrm{i}}} q_{\mathrm{v} r}(0) \\
G_{v}(D) & =4 \frac{1-\rho^{\mathrm{o}}}{1-\rho^{\mathrm{i}}} q_{\mathrm{rr} 2}^{\mathrm{o}}+2 \frac{1+\rho^{\mathrm{i}}}{1-\rho^{\mathrm{i}}} q_{\mathrm{rr}}(D) .
\end{aligned}
$$

By using equations (8) and (11a), and equations (9) and $(1 \mathrm{lb})$, the $T(0)$ and $T(D)$ are eliminated and the following relations are obtained by solving the two remaining equations for the integration constant $\operatorname{CON}$, and for $q_{\mathrm{tot}}$,

$$
\begin{aligned}
\operatorname{CON}= & {\left[\frac{h_{1}}{k}\left(1+\frac{h_{2}}{h_{1}}+\frac{h_{2} D}{k}\right)\right]^{-1}\left\{h_{1}\left(1+\frac{h_{2} D}{k}\right)\right.} \\
& \times\left[T_{\mathrm{g} 1}+\frac{1}{3 k} \int_{0}^{\cdot} \frac{G_{v}(0)}{K_{v}} \mathrm{~d} v\right] \\
& +h_{2}\left[T_{\mathrm{v} 2}+\frac{1}{3 k} \int_{0}^{x} \frac{G_{v}(D)}{K_{v}} \mathrm{~d} v\right] \\
& \left.+\left(1+\frac{h_{2} D}{k}\right) \int_{0}^{x} q_{\mathrm{v}}(0) \mathrm{d} v-\int_{0}^{x} q_{\mathrm{vr}}(D) \mathrm{d} v\right\} \\
q_{\mathrm{t}+1}= & h_{1}\left[T_{\mathrm{z} 1}+\frac{1}{3 k} \int_{0}^{x} G_{v}(0) \mathrm{d} v-\frac{C O N}{k}\right] \\
K_{\mathrm{v}} & +\int_{0}^{x} q_{\mathrm{vr}}(0) \mathrm{d} v .
\end{aligned}
$$

Equations in dimensionless form for a gray layer. The equations for multiple spectral bands follow quite readily from the preceeding relations and from relations for a gray layer (one spectral band). The gray relations are now given in dimensionless form using quantities defined in the Nomenclaturc.

Two-flux equations :

$$
\tilde{q}_{r}(X)=\tilde{q}_{r}(0)+\kappa_{1}(1-\Omega) \int_{0}^{x}\left[4 n^{2} t^{4}(X)-\tilde{G}(X)\right] \mathrm{d} X
$$

$$
\tilde{G}(X)=\tilde{G}(0)-3 \kappa_{1} \int_{0}^{x} \tilde{q}_{t}(X) \mathrm{d} X
$$

Energy equation :

$$
l(X)=\frac{1}{N}\left[-\frac{\tilde{G}(X)}{3 \kappa_{\mathrm{D}}}-\tilde{q}_{\mathrm{m}} X+C \tilde{O} N\right] .
$$

Equations for $\tilde{G}(0)$ and $\tilde{G}(D)$ :

$$
\begin{aligned}
& \tilde{G}(0)=4 \frac{1-\rho^{\circ}}{1-\rho^{i}} \tilde{q}_{r l}^{\prime \prime}-2 \frac{1+\rho^{\prime}}{1-\rho^{\prime}} \tilde{q}_{r}(0) \\
& \tilde{G}(1)=4 \frac{1-\rho^{\prime \prime}}{1-\rho^{i}} \tilde{q}_{r 2}^{o}+2 \frac{1+\rho^{\prime}}{1-\rho^{i}} \bar{q}_{r}(1) .
\end{aligned}
$$

Equations for $C \tilde{O} N$ and $\tilde{q}_{1 o 1}$ :

$$
\begin{aligned}
& C \tilde{O} N=\left[\frac{H_{1}}{N}\left(1+\frac{H_{2}}{H_{1}}+\frac{H_{2}}{N}\right)\right]^{-1}\left\{H_{1}\left(1+\frac{H_{2}}{N}\right)\right. \\
& \times\left[1+\frac{\tilde{G}(0)}{3 N \kappa_{D}}\right]+H_{2}\left[t_{\mathrm{g} 2}+\frac{\tilde{G}(1)}{3 N \kappa_{\mathrm{D}}}\right] \\
& \left.+\left(1+\frac{H_{2}}{N}\right) \tilde{q}_{r}(0)-\tilde{q}_{r}(1)\right\} \\
& \tilde{q}_{101}=H_{1}\left[1+\frac{\tilde{G}(0)}{3 N \kappa_{1)}}-\frac{C \tilde{O} N}{N}\right]+\tilde{q}_{r}(0) \text {. }
\end{aligned}
$$

Solution procedure by iteration for a gray layer. An iterative solution procedure was used by guessing valucs of the dimensionless boundary temperatures $t(0)$ and $t(1)$. The boundary relations given by equations $(19 \mathrm{a}, \mathrm{b}),(18)$ at $X=0$ and 1 , and $(11 \mathrm{a}, \mathrm{b})$ in dimensionless form for a gray layer, are solved simultaneously to find starting values for $\tilde{q}_{r}(0)$ and $\bar{q}_{r}(1)$ and $\tilde{q}_{\mathrm{|a|}}$. A $\tilde{q}_{\mathrm{r}}(X)$ distribution passing through the end values and through $\tilde{q}_{\text {tot }}$ at $X=1 / 2$ was used as an approximation to start the iteration. Using $\tilde{G}(0)$ and $\tilde{G}(1)$ calculated from equations (19a.b) the trial $\bar{q}_{\mathrm{r}}(X)$ is normalized such that the condition $\left.3 \kappa_{1}\right) \int_{11}^{1} \tilde{q}_{1}(X) \mathrm{d} X=\tilde{G}(0)-\tilde{G}(1)$ from equation (17) is satisfied. The $\tilde{G}(X)$ is then obtained from equation (17), and $C \tilde{O} N$ and $\bar{q}_{101}$ are found from equations (20) and (2I). The $f(X)$ is evaluated from equation (18) and a new $\tilde{q}_{r}(X)$ is obtained from equation (16). To begin a new itcration a damping factor is applied between the new and old $\tilde{q}_{r}(X)$ to keep the iterative method stable. A small damping factor was needed such as 0.0005 when the optical thickness is about 10 and there is no scattering. Solutions with scattering required less damping. Computing time for a solution converged to a maximum error on $\bar{q}_{\mathrm{r}}(X)$ of $5 \times 10^{3}$ depends on the integration method used in equations (16) and (17). Solutions using a trapezoidal rule took seconds on a VAX computer. A Gaussian routine took from seconds to minutes depending on the optical properties and gave comparable results to the trapezoidal rulc.

The surface reflection characteristics were modeled by using integrated averages of the Fresnel reflection relations. For diffuse incident radiation this gives $[6$. p. 115], 


$$
\begin{aligned}
\rho(n) \equiv & R(n)=\frac{1}{2}+\frac{(3 n+1)(n-1)}{6(n+1)^{2}} \\
& +\frac{n^{2}\left(n^{2}-1\right)^{2}}{\left(n^{2}+1\right)^{3}} \ln \left(\frac{n-1}{n+1}\right) \\
& -\frac{2 n^{3}\left(n^{2}+2 n-1\right)}{\left(n^{2}+1\right)\left(n^{4}-1\right)}+\frac{8 n^{4}\left(n^{4}+1\right)}{\left(n^{2}+1\right)\left(n^{4}-1\right)^{2}} \\
& \times \ln (n) \quad\left(n=n_{\mathrm{h}} / n_{\mathrm{s}}\right) .
\end{aligned}
$$

Equation (22) is for reflection of radiation incident on a material of higher refractive index where $n_{\mathrm{h}}$ and $n_{\mathrm{s}}$ are the 'higher' and 'smaller' $n$ values (the incident radiation is from within the material with $n_{s}$ ). After allowing for energy incident at angles larger than the critical angle for total reflection, the $\rho(n)$ for diffuse radiation going in the direction from a higher to a smaller refractive index material is found from [9].

$$
\rho(n)=1-\frac{1}{n^{2}}[1-R(n)] \quad\left(n=n_{\mathrm{h}} / n_{\mathrm{s}}\right)
$$

Two-flux method for a two-band spectral calculation. A two-band calculation is used to illustrate a spectral application of the two-flux method. The letters $S$ and $L$ designate the ranges with small and large frequencies. Then for a quantity such as $G_{v}(0)$ the band notation is used that $G S(0)=\int_{0}^{r_{s}} G_{r}(0) d v$ and $G L(0)=\int_{v_{i}}^{x} G_{r}(0) \mathrm{d} v$. For a two-band calculation each of equations (19) has two parts, one for each frequency range. For example, using equation (19a) gives for $\widetilde{G}(0)$ in the small and large frequency ranges,

$$
\begin{aligned}
& \tilde{G} S(0)=4 \frac{1-\rho^{\circ}}{1-\rho^{\mathrm{i}}} \tilde{q} S_{\mathrm{r} 1}^{\circ}-2 \frac{1+\rho^{\mathrm{i}}}{1-\rho^{\mathrm{i}}} \tilde{q} S_{\mathrm{r}}(0) \\
& \tilde{G} L(0)=4 \frac{1-\rho^{\mathrm{o}}}{1-\rho^{\prime}} \tilde{q} L_{\mathrm{r} 1}^{\circ}-2 \frac{1+\rho^{\mathrm{i}}}{1-\rho^{\prime}} \tilde{q} L_{\mathrm{r}}(0) .
\end{aligned}
$$

Similar relations are written from equation (19b) for $\tilde{G} S(1)$ and $\tilde{G} L(1)$. Equation (21) for $\tilde{q}_{\mathrm{tot}}$ now contains a contribution from each of the two bands,

$$
\begin{aligned}
\tilde{q}_{1 \mathrm{Lt}}=H_{1}\left[1+\frac{\tilde{G} S(0)}{3 N h_{\mathrm{DS}}}+\frac{\tilde{G} L(0)}{3 N \kappa_{\mathrm{DL}}}-\frac{C \tilde{O} N}{N}\right] \\
+\bar{q} S_{\mathrm{r}}(0)+\dot{q} L_{\mathrm{r}}(0) .
\end{aligned}
$$

This also occurs in the temperature relation equation (18).

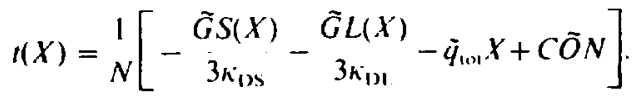

Equation (16) for the radiative flux is written for each band. For the band with short frequencies.

$$
\begin{aligned}
\tilde{q} S_{\mathrm{r}}(X)=\tilde{q} S_{\mathrm{r}}(0) & +\kappa_{\mathrm{DSS}}\left(1-\Omega_{\mathrm{r}}\right) \\
& \times \int_{11}^{1}\left[4 n^{2} t^{+}(X) F S(X)-\tilde{G} S(X)\right] \mathrm{d} X
\end{aligned}
$$

and similarly for $\bar{q} L_{\mathrm{r}}(X)$ in the band with large fre- quencies. The $F S(X)$ is the fraction of blackbody energy in the short frequency range corresponding to the temperature $t(X) T_{\mathrm{gt}}$.

The solution by iteration is similar to that outlined for a gray layer except there are now two $\dot{q}_{\mathrm{r}}(X)$, one for each spectral band. The iteration begins by estimating $\tilde{q} S_{\mathrm{r}}(X)$ and $\tilde{q} L_{\mathrm{r}}(X)$ by taking into account that $\tilde{q}_{\mathrm{r}}(X)$ tends to be small in a band where $\kappa_{D}$ is large and that the radiative energy distribution in the bands shifts to smaller $v$ as $t(X)$ decreases.

The diffusion approximation for optically thick spectral layers

When the optical thickness of a layer is large the radiative diffusion method is very useful for making predictions using spectrally dependent properties. Since the diffusion method yields equations that are convenient to evaluate, the method is extended here from a single layer to a multilayer laminated composite of $J$ layers as shown in Fig. 2. From ref. [6, p. 753], the radiative flux by diffusion in any of the layers is given by $\mathrm{d} q_{\mathrm{rr}}=-\left(4 / 3 K_{\mathrm{v}}\right)\left(\mathrm{d} e_{\mathrm{vb}} / \mathrm{d} x\right) \mathrm{d} v$. To obtain the radiative heat flux including all frequencies this is integrated over all $v$ to give

$$
q_{r}=-\frac{4}{3} \frac{\mathrm{d}}{\mathrm{d} x} \int_{0}^{x} \frac{e_{r b}}{K_{r}} \mathrm{~d} v
$$

For integrating over all $v$ it is convenient to use multiple spectral bands where $K_{\mathrm{r}}=K_{m}$ in the $m$ th band and there is a total of $M$ bands. Then with each band extending from $v_{m}$ to $v_{m+1}$ where $1 \leqslant m \leqslant M$,

$$
q_{\mathrm{r}}=-\frac{4}{3} \sum_{m=1}^{M} \frac{1}{K_{m}} \frac{\mathrm{d}}{\mathrm{d} x} \int_{v_{m}}^{r_{m+1}} e_{\mathrm{vb}}(v) \mathrm{d} v
$$

The quantity in the integral can be expressed in terms of the blackbody fraction $F\left(v / c_{0} T\right)$,

$$
\int_{r_{m}}^{v_{m+1}} e_{m}(v) d v=n^{2} \sigma T^{4}\left[F\left(v_{m+1} / c_{11} T\right)-F\left(v_{m} / c_{0} T\right)\right] .
$$

The total energy transfer by radiation and conduction is then given by,

$$
\begin{aligned}
q_{(t) 1}= & -\left[k \frac{\mathrm{d} T}{\mathrm{~d} x}+\frac{4}{3} \sum_{m=1}^{M} 1\right. \\
& \left.\times \frac{\mathrm{d}}{\mathrm{d} x} K_{m} n^{2} \sigma T^{4}\left[F\left(v_{m+1} / c_{0} T\right)-F\left(v_{m} / c_{0} T\right]\right\}\right]
\end{aligned}
$$

Since $q_{1,1}$ is a constant through the composite layer equation (31) can be integrated from 0 to $x_{j}$ in the $j$ th layer. The result is placed in dimensionless form using the quantities defined in the Nomenclature to yield,

$$
\begin{aligned}
& \tilde{y}_{10 t} X_{1}=N_{i}\left[t_{, i}-t_{i}\left(X_{i}\right)\right]+\frac{4 n_{i}^{2}}{3} \\
& \times \sum_{m=1}^{M} \frac{1}{K_{m} D_{i}}\left[t _ { i , r } ^ { 4 } \left[F\left(v_{m+1} / c_{n, 1, i} T_{m !}\right)\right.\right.
\end{aligned}
$$




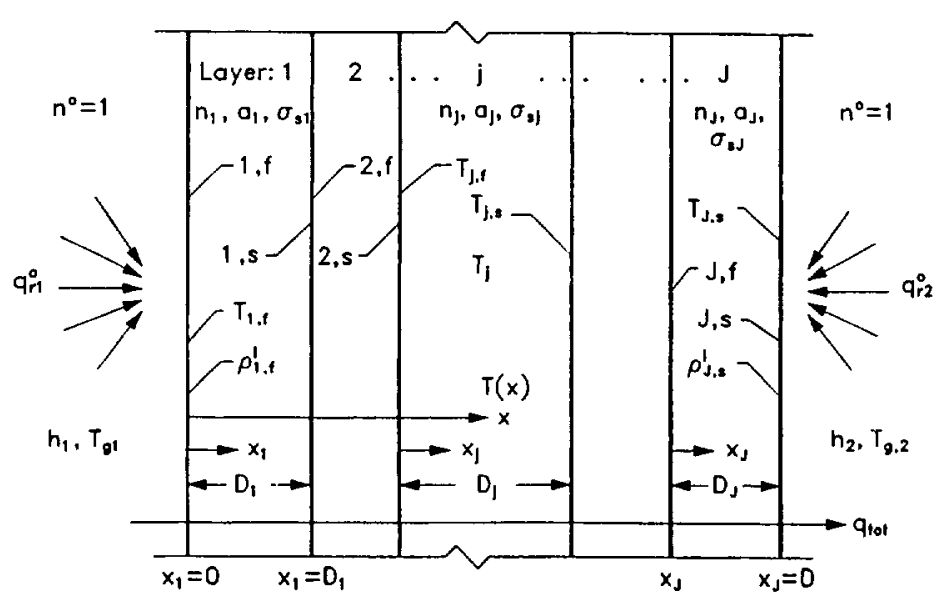

FIG. 2. Laminated multilayered geometry showing coordinate system and nomenclature designating interfaces and their temperatures for use in spectral diffusion method for single or multiple layered composites.

$$
\begin{aligned}
& \left.-F\left(v_{m} / c_{0} l_{j, \mathrm{r}} T_{\mathrm{g} 1}\right)\right\}-t_{j}^{4}\left(X_{j}\right)\left\{F\left[v_{m+1} / c_{0} t_{j}\left(X_{j}\right) T_{\mathrm{g} 1}\right]\right. \\
& \left.\left.-F\left[v_{m} / c_{0} t_{j}\left(X_{j}\right) T_{\mathrm{g} 1}\right]\right\}\right]
\end{aligned}
$$

where $t_{j, \Omega} \equiv t_{i}\left(X_{i}=0\right)$ is at the first interface in the $j$ th layer (see Fig. 2).

Equation (32) is evaluated at $X_{j}=1$ which gives an expression for the temperature difference across the $j$ th layer in terms of $\tilde{q}_{10}$. To join two layers a continuous temperature is assumed at the internal interface between them. The temperature jump that occurs in some instances at an interface when using the diffusion approximation is neglected at the boundaries. The jump is small when the layers are optically thick and when conduction is comparable to radiation [10]. The convective and radiative balance at each outside boundary, and the conduction with radiative diffusion relation for $\tilde{q}_{\mathrm{tol}}$, equation (32), written for the temperature difference across each of the $J$ layers, provide the following simultaneous equations :

$$
\begin{aligned}
& H_{1}\left(1-t_{1 . f}\right)+\left(1-\rho_{1 . f}^{i}\right) n_{i}^{i}\left[\tilde{q}_{t 1}^{0}-t_{1 . f}^{4}\right]-\tilde{q}_{101}=0 \\
& \tilde{q}_{\mathrm{tot}}=N_{i}\left[t_{j, \mathrm{f}}-t_{i . \mathrm{j}}\right]+\frac{4 n_{i}^{2}}{3} \\
& \times \sum_{m=1}^{M} \frac{1}{K_{m} D}\left[t _ { i . \mathrm { f } } ^ { 4 } \left\{F\left(v_{m+1} / c_{0} t_{, \mathrm{r}} T_{\mathrm{g} 1}\right)\right.\right. \\
& \left.-F\left(v_{m} / c_{a} t_{i, f} T_{k 1}\right)\right\}-t_{i, s}^{4}\left\{F\left[v_{m+1} / c_{0} t_{j, s} T_{\mathrm{g} 1}\right]\right. \\
& \left.-F\left[v_{m} / c_{0} t_{j, \mathrm{~s}} T_{\mathrm{g} 1}\right]^{\prime}\right] \quad(1 \leqslant j \leqslant \Omega) \\
& H_{2}\left(t_{J, \mathrm{~s}}-t_{\mathrm{g} 2}\right)+\left(1-\rho_{J, \mathrm{~s}}^{\mathrm{i}}\right) n^{2}\left(t_{J_{\mathrm{S}}}^{4}-\tilde{q}_{\mathrm{r} 2}^{\mathrm{o}}\right)-\dot{q}_{1 \mathrm{ol}}=0 \text {. }
\end{aligned}
$$

These equations are solved for the two external boundary temperatures $t_{1 . f}$ and $t_{i, s}$, the internal interface temperatures, and the heat flow $\tilde{q}_{\text {tol }}$ through the composite layer. After $\bar{q}_{101}$ is obtained, the temperature distribution in each layer $(1 \leqslant j \leqslant J)$ is calculated by solving equation (32) numerically for $t,(X$,$) .$
Hybrid method using the two-flux method for thin bands and the diffusion method for thick bands

The hybrid energy equation. In the numerical solutions using the two-flux method, convergence was found more difficult for a layer with optical thickness larger than about 20. To analyze spectral cases with both optically thin and thick bands, a hybrid method was devised. For optically thin bands the two-flux method is used, while the diffusion method is used for thick bands. For simplicity a two-band model is given here where the optically thick region is for $0 \leqslant v \leqslant v$. so that $\kappa_{\mathrm{DS}}>\kappa_{\mathrm{DL}}$. The total flux by conduction and radiation is

$$
q_{1 \mathrm{ol}}=-k \frac{\mathrm{d} T}{\mathrm{~d} x}+\int_{v=0}^{v_{\mathrm{v}}} q_{v \mathrm{r}} \mathrm{d} v+\int_{v_{\mathrm{c}}}^{\infty} q_{v \mathrm{r}} \mathrm{d} v .
$$

Using the diffusion relation from equation (28) for the 1 st integral and the two-flux relation, equation (4), for the 2 nd integral gives

$$
\begin{aligned}
q_{101}=-k \frac{\mathrm{d} T(x)}{\mathrm{d} x}-\frac{4}{3 K_{\mathrm{S}}} \frac{\mathrm{d}}{\mathrm{d} x} \int_{0}^{\mathrm{c}_{\mathrm{c}}} e_{\mathrm{rb}}(x) \mathrm{d} v \\
\\
-\frac{1}{3 K_{\mathrm{L}}} \frac{\mathrm{d}}{\mathrm{d} x} \int_{r}^{x} G_{r}(x) \mathrm{d} v .
\end{aligned}
$$

By integrating equation (35) and putting it in dimensionless form the temperature distribution becomes

$$
\begin{aligned}
t(X)=\frac{1}{N}\left\{-\frac{4}{3 \kappa_{\mathrm{DS}}} t(X)^{4} F S[t(X)]\right. \\
\left.\quad-\frac{\tilde{G} L(X)}{3 \kappa_{\mathrm{DL}}}-\dot{q}_{\mathrm{to}} X+C \tilde{O} N\right\}
\end{aligned}
$$

as compared with equation (26) using the two flux method for both spectral bands. Since $r(X)$ in equation (36) is in the blackbody fraction $F S[r(X)]$, a numerical 
method such as a root solver is used to find the distribution $t(X)$.

Boundary conditions. For the diffusion approximation. external radiative energy in the optically thick band is absorbed and emitted at the boundary surface at $x=0$. The net external energy that is absorbed, combined with the external convection, is equal at the boundary to the internal heat conduction combined with the internal radiative diffusion (which acts like conduction) in the optically thick band (small v). The total internal heat flow at the boundary can then be expressed as this net external energy (absorbed radiation and convection) added to the internal radiation in the optically thin band (large $v$ ) which is obtained from the two-flux method,

$$
\begin{aligned}
q_{1 \mathrm{ol}}=\left(1-\rho^{\mathrm{v}}\right)\left\{q S_{\mathrm{r} 1}^{\mathrm{o}}-\right. & \left.\sigma T(0)^{4} F S[T(0)]\right\} \\
& +h_{1}\left[T_{\mathrm{g} 1}-T(0)\right]+q L_{\mathrm{r}}(0) .
\end{aligned}
$$

Similarly at $x=D$,

$$
\begin{aligned}
q_{\mathrm{tot}}=-\left(1-\rho^{\circ}\right)\left\{q S_{\mathrm{r} 2}^{\circ}-\sigma T(D)^{4} F S[T(D)]\right\} \\
+h_{2}\left[T(D)-T_{\mathrm{z}^{2}}\right]+q L_{\mathrm{r}}(D) .
\end{aligned}
$$

From equation (36) in dimensional form evaluated at $x=0$ and $D$,

$$
\operatorname{CON}=k T(0)+\frac{4}{3 K_{\mathrm{S}}} T(0)^{4} F S[T(0)]+\frac{1}{3 K_{\mathrm{L}}} G L(0)
$$

$$
\begin{aligned}
q_{101}= & \frac{C O N}{D}-\frac{k}{D} T(D) \\
& -\frac{4}{3 K_{\mathrm{S}} D} T(D)^{4} F S[T(D)]-\frac{1}{3 K_{\mathrm{l}} D} G L(D) .
\end{aligned}
$$

Combine equations (37a) and (38a) to climinate the $T(0)$ term, and combine cquations (37b) and (38b) to eliminate the $T(D)$ term. Then eliminate $q_{101}$ from the resulting two equations. This yields the equation for $C O N$ in dimensionless form (as in equation (20)) as

$$
\begin{aligned}
& r \tilde{O N}=\left[\frac{H_{1}}{N}\left(1+\frac{H_{2}}{H_{1}}+\frac{H_{2}}{N}\right)\right]^{-1}\left\{H_{1}\left(1+\frac{H_{2}}{N}\right)\right. \\
& \times\left[1+\frac{\tilde{G} L(0)}{3 N_{\kappa_{1 \mathrm{~L}}}}+\frac{4}{3 N_{\kappa_{\mathrm{DSS}}}}(0)^{4} F S[t(0)]\right] \\
& +H_{2}\left[t_{\mathrm{y}_{2}}+\frac{\tilde{G} L(1)}{3 N_{k_{\mathrm{DI}}}}+\underset{3 N_{\mathrm{h}_{\mathrm{DS}}}}{4}(1)^{4} F S[t(1)]\right] \\
& +\left(1+\begin{array}{c}
H_{2} \\
N
\end{array}\right)\left(\dot{q} L_{\mathrm{r}}(0)+\left(1-\rho^{\prime \prime}\right)\right. \\
& \times\left[\bar{q} S_{\mathrm{r} 1}^{0}-t(0)^{4} F S[t(0)]\right]_{i}-\bar{q} L_{\mathrm{r}}(1)+\left(1-\rho^{\prime \prime}\right) \\
& \times\left[\tilde{q} S_{\mathrm{r}_{2}}^{\mathrm{o}}-t(1)^{+} F S[t(1)]\right]_{i}^{\prime} \text {. }
\end{aligned}
$$

The $\dot{q}_{\mathrm{tm}}$ can be obtained from from either equation (37) or (38b).

Solution method. The itcrative solution starts by assuming $\tilde{q} L(X) . t(0)$ and $t(D)$. The $\tilde{C} L(0)$ is found from equation (24b) and similarly for $\tilde{G} L(1)$. The $q L_{r}(X)$ is normalized according to the two-flux equation (17) so that $3 k_{\mathrm{D} L} \int_{0}^{1} \tilde{q} L_{\mathrm{r}}(X) \mathrm{d} X=\tilde{G} L(0)-\tilde{G} L(1)$. Then $G L(X)$ is found from equation (17) as

$$
\tilde{G} L(X)=\tilde{G} L(0)-3 \kappa_{\mathrm{DL}} \int_{0}^{x} \tilde{q} L_{\mathrm{r}}(X) \mathrm{d} X .
$$

The $C \tilde{O} N$ is obtained from equation (39), and $\tilde{q}_{\text {tot }}$ from equations (37) or (38b). The $t(X)$ is then obtained from equation (36), and a new $\tilde{q} L_{r}(X)$ is computed from the two-flux equation (27) written for the large $v$ band. To start the next iteration a damping factor is applied between this $\tilde{q} L_{r}(X)$ and the $\dot{q} L_{r}(X)$ that was used to start the iteration.

\section{RESULTS AND DISCUSSION}

\section{Two-flux results for a gray layer}

The most basic application of the two-flux method is for a gray layer. Two-flux results are given in refs. $[7,8]$ for a layer with a refractive index $n=1$ between two black walls at specified temperatures. Excellent agreement was obtained with numerical solutions in the literature of the exact radiative transfer equations. In the present work the boundary temperatures are unknown and are obtained in the solution as a result of specified convection and incident radiation conditions at the boundaries. The present results show the effect of having a refractive index as large as $n=4$ and the effect of isotropic scattering with an albedo of $\Omega=0.9$.

Figure 3 is for $n=2$, and Fig. 3(a) is without scattering. The 'exact' numerical results were obtained in refs. [2.3] where the behavior of the temperature distributions was discussed. The two-flux method yields excellent predictions of the temperature distributions and the results follow the pronounced temperature curvature near the boundaries. There are only small deviations from the exact solution; the largest deviations are for $\kappa_{D}=1$ where radiation has a large effect since the layer is neither optically thin or thick. The total heat flux through the layer by combined radiation and conduction is predicted very well by the two-flux method as shown on the figure.

The effect of large scattering, $\Omega=0.9$, in Fig. 3(b) is for the same $n$ and $\kappa_{D}$ values as in Fig. 3(a). For the same $\kappa_{0}$ a layer with $\Omega=0.9$ has $10 \%$ of the absorption as for $\Omega=0$. For these conditions the twoflux relations provide excellent predictions for all $\kappa_{\mathrm{D}}$ of both the temperature distribution and total energy transfer through the layer

When the refractive index is increased to $n=4$, with all other parameters kept the same as in Figs. 3(a),(b), the results are in Figs. 4(a),(b). The exact numerical solutions are again obtained from refs. $[2,3]$. The temperature profiles are more uniform in the central portion of the layer as discussed in refs. [2, 3] which leads to large temperature variations near the boundaries. The two-flux method is successful in predicting both the details of the temperature distributions and total 

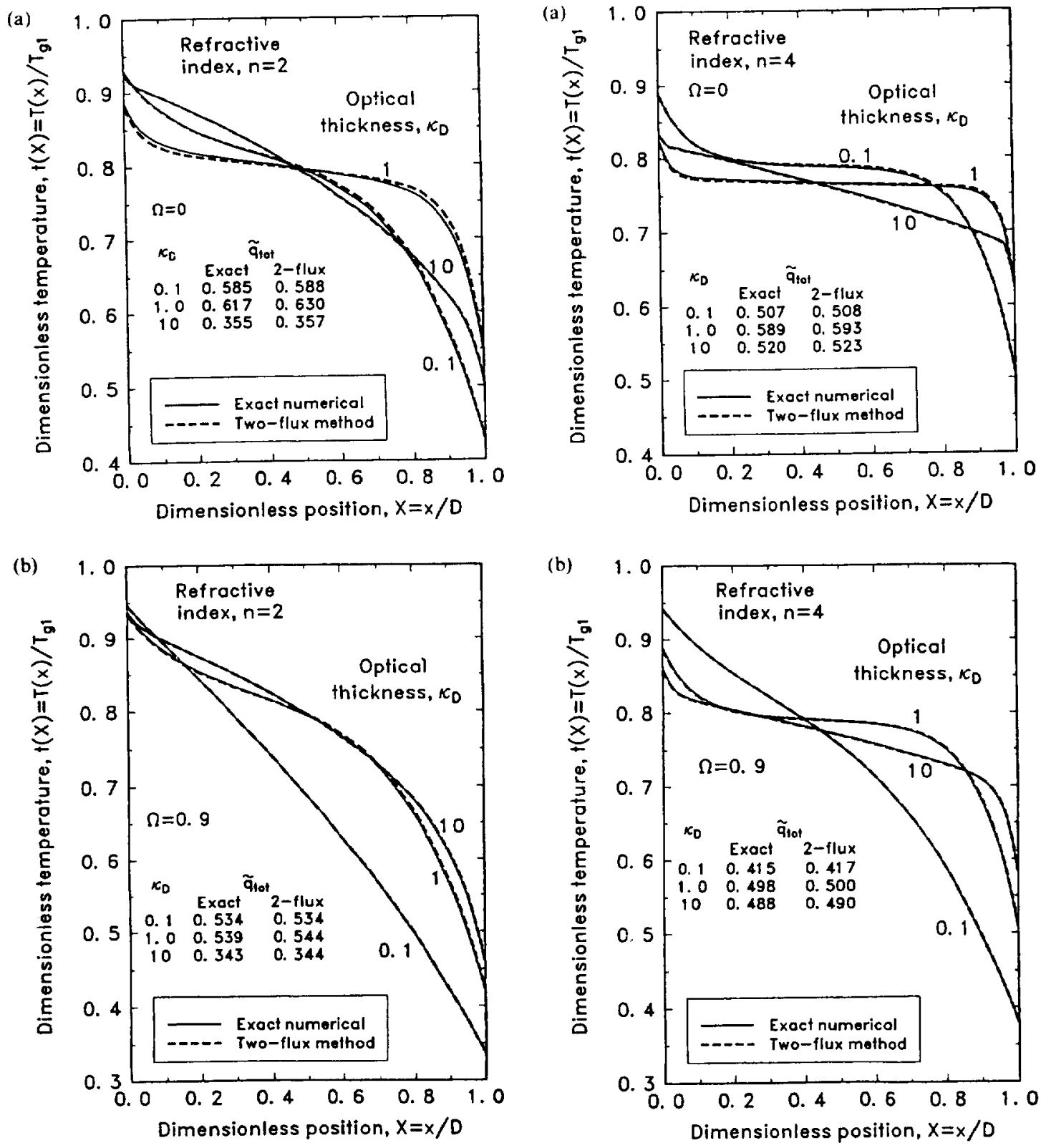

FjG. 3. Comparisons of two-flux results with exact numerical solutions of radiative transfer equations without and with scattering in a gray layer with refractive index $n=2 ; \bar{q}_{r}^{*}$ $=1, \tilde{q}_{2}^{\circ}=0.25^{4}, t_{k 1}=1, t_{k 2}=0.25, H_{1}=H_{2}=1, N=0.1$. (a) Scattering albedo, $\Omega=0$. (b) Scattering albedo, $\Omega=0.9$.

heat flux transferred by combined radiation and conduction.

Two-flux results for a layer with wo absorption hands

For the results in Fig. 5(a) the spectral absorption coefficient has two values : in the small $v$ range $\left(v / c_{0} T_{\mathrm{g}}\right.$ $<1 / 4000), a_{r s} D=10$, while in the large $v$ range $\left(v_{c} / c_{0} T_{\mathrm{g} 1}>1 / 4000\right), a_{\mathrm{r}} D=0.1$. The selected value of the cutoff frequency $v_{c}$ divides the black body spectrum at $T_{\mathrm{g} 1}$ into approximately two equal parts. A gray scattering component is present so that for the two

Fig. 4. Comparisons of two-flux results with exact numerical solutions of radiative transfer equations without and with scattering in a gray layer with refractive index $n=4: \bar{q}_{i 1}^{\prime \prime}$ $=1, a_{r 2}^{\prime \prime}=0.25^{4}, t_{x 1}=1, t_{R 2}=0.25, H_{1}=H_{2}=1, N=0.1$. (a) Scattering albedo, $\Omega=0$. (b) Scattering albcdo, $\Omega=0.9$.

temperature profiles in Fig. 5(a), $\sigma_{,} D=0.9$ or $\sigma, D=9.9$. Thus for the (wo curves the spectral optical thicknesses are, respectively, $\kappa_{12 S}=10.9 . \kappa_{1)}=1$, and $\kappa_{\mathrm{DS}}=19.9, \kappa_{\mathrm{DL}}=10$. As shown the two-flux method applied spectrally, as developed in the analysis, provides excellent agreement with the two-band numerical solutions in ref. [3].

In Fig. 5(b) the $a_{v} D$ values are increased to 100 and 1 for the bands with small and large frequencies, and the gray scattering component is $\sigma, D=9$. The spectral optical thicknesses are then $\kappa_{\mathrm{DS}}=109$ and $\kappa_{\mathrm{DL}}=10$. 

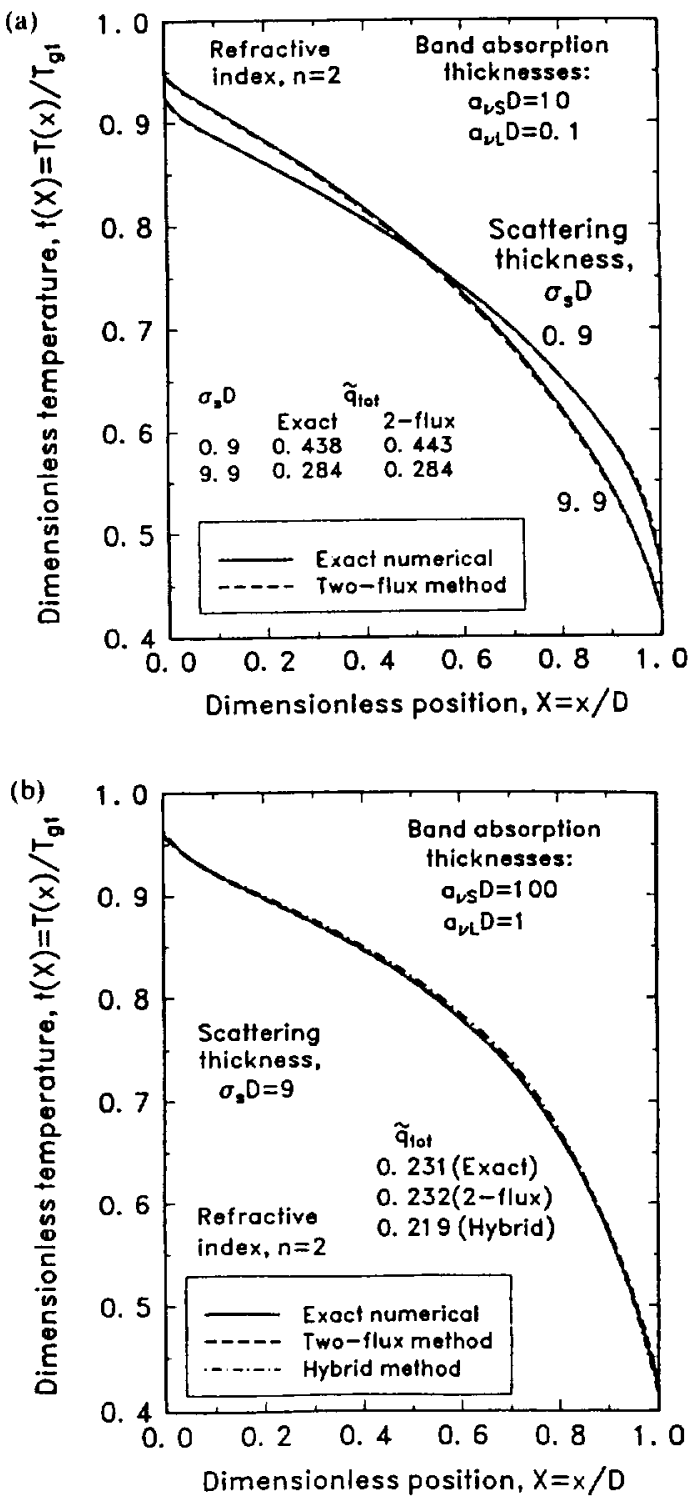

Fici. 5. Comparisons of two-flux and hybrid results with exact numerical solutions of radiative transler equations for two-band spectral calculations with scattering and i refractive index of $n=2: \bar{q}_{11}^{\prime \prime}=1 . \tilde{q}_{i 2}^{\prime \prime}=0.25^{4}, t_{t 1}=1, t_{0}=0.25$ $H_{1}=H_{2}=1, N=0.1$, ${ }_{2} c_{01} T_{\mathrm{k}}=1 / 4000$. (a) Band absorplion thicknesses : $a_{r \mathrm{~s}} D=10, a_{\mathrm{vi}} D=0.1$. (b) Band absorption thicknesses : $a_{\mathrm{r} s} D=100, a_{\mathrm{rl}} D=1$.

The two-flux method provides excellent agreement of $t(X)$ and $\bar{q}_{\text {ot }}$ with the exact numerical solution of the transfer equations from ref. [3]. Results from the hybrid method are also shown and they provide very good agreement. For a large $\kappa_{1}$ in one band the hybrid method provides more rapid convergence than the two-flux method.

Spectral diffiusion results for single or multiple layers

Temperature distributions are shown in Fig. 6 for a layer with refractive index $n=2$ and three spectral bands within which the optical thickriesses are 5. 10.

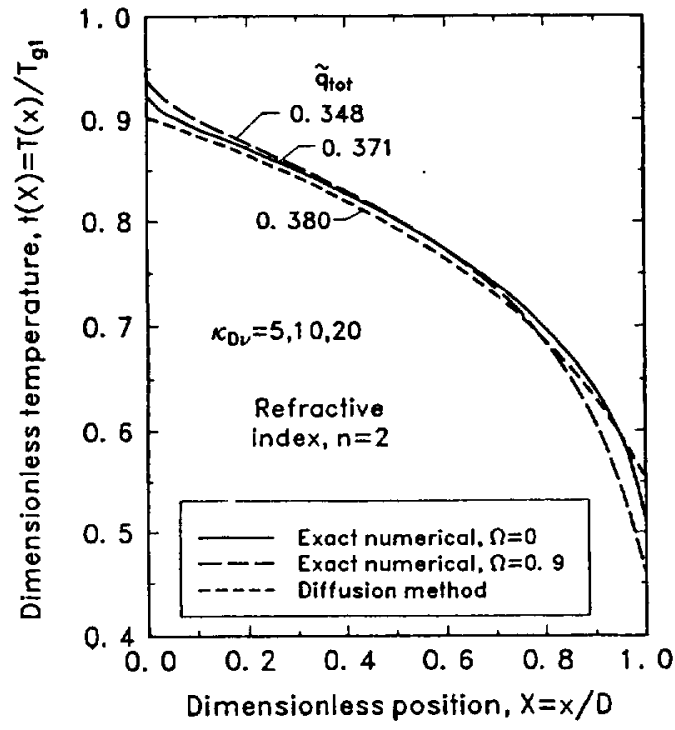

FiG. 6. Comparison of spectral diffusion method predictions with temperature profiles from exact numerical solution of the radiative transfer equations for a layer with three spectral bands without scattering and with an albedo of $0.9 ; \tilde{q}_{11}^{\mathrm{o}}=1$. $\tilde{q}_{\mathrm{r} 2}^{\mathrm{o}}=0.25^{4}, \quad t_{\mathrm{z} 1}=1, \quad t_{\mathrm{k} 2}=0.25, \quad H_{1}=H_{2}=1, \quad N=0.1$. Optical thicknesses in the three bands in order of increasing frequency are $\kappa_{\mathrm{Dr}}=5,10,20$, and the two cutoff frequencies are given by $v_{\mathrm{c}} / c_{0} T_{\mathrm{g} 1}=1 / 5300,1 / 3300$.

and 20 in order of increasing frequency. The two cutoff frequencies (given in the caption) divide blackbody radiation at $T_{\mathrm{g}}$ into approximately three equal parts. Two exact numerical solutions are given: the solid line is without scattering $(\Omega=0)$ while the long dash line is for $\Omega=0.9$. The diffusion approximation is independent of the amount of scattering and hence only one diffusion curve is given (short dashed line). The diffusion curve is close to the other curves but does not follow their exact shape near the boundaries. This is expected as the diffusion approximation (based on an isotropic assumption) does not apply adjacent to a boundary because the flux in this region is not isotropic. The diffusion method predicts the heat transfer through the layer within $10 \%$.

The diffusion method provides a very good prediction of the temperature distribution in a three layer region as shown in Fig. 7. The exact numerical solution was obtained by cxtending the computer program from ref. [4]. Each layer has three spectral bands defined in the caption. The diffusion results are considerably casicr to compute than the numerical solution of the radiative transfer equations for three coupled layers and three absorption bands. The opaque limit for conduction (no radiation) within the layers, and with emission, absorption, and convection at the outer boundaries is shown as a dot-dash line. This shows that there is a substantial internal radiation effect although the combined optical thicknesses through the three layers is moderately large. A good prediction (within $4 \%$ ) is obtained for the heat transfer through the composite. 


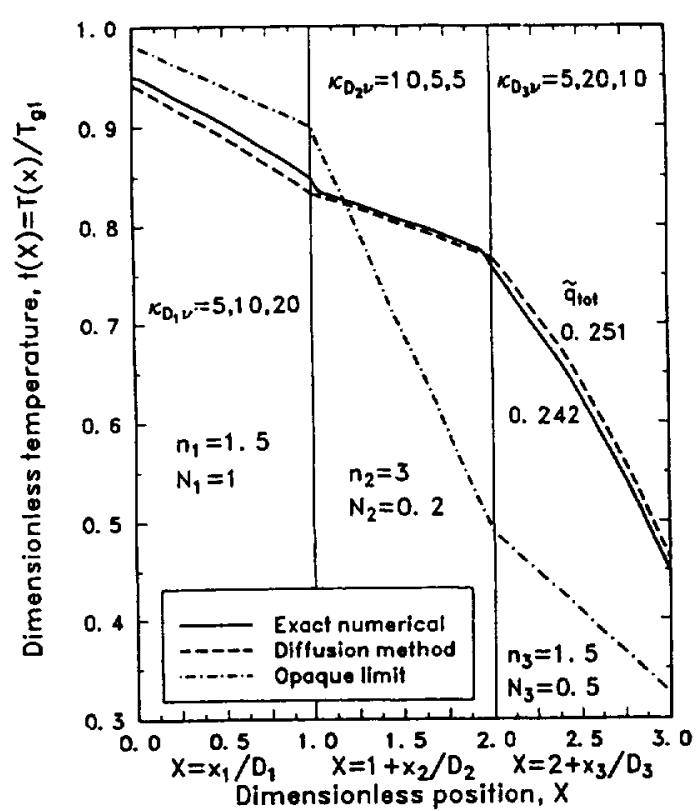

FIG. 7. Comparison of spectral diffusion method predictions with temperature profiles from the exact numerical solution of the radiative transfer equations for a three-layer composite with three spectral bands; $\tilde{q}_{n 1}^{o}=1, \tilde{q}_{r 2}^{o}=0.25^{4}, t_{s 1}=1$, $t_{\mathrm{s} 2}=0.25, H_{1}=H_{2}=1$. Optical thicknesses in the three spectral bands in order of increasing frequency are $\kappa_{D_{, r}}=5,10,20, \kappa_{D_{2}}=10,5,5, \kappa_{D, v}=5,20,10$, and the two cutoff frequencies are given by $v_{d} / c_{0} T_{a 1}=1 / 8000,1 / 4000$.

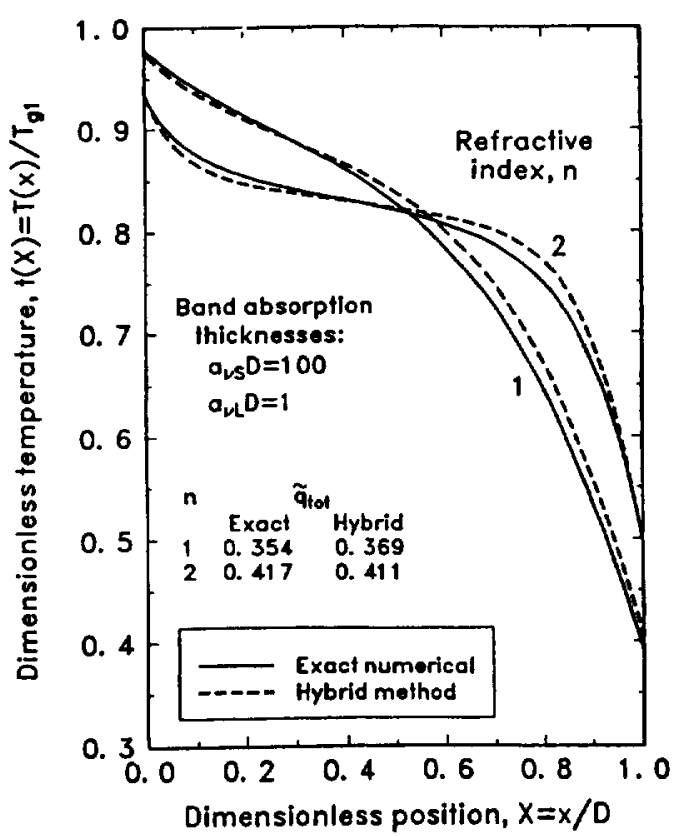

Fig. 8. Comparison of hybrid two-flux/diffusion method with the exact numerical solution for a layer with one optically thin spectral band $\left(\kappa_{\mathrm{DL}}=1\right)$, and one optically thick band $\left(\kappa_{\mathrm{Ds}}=100\right) ; \tilde{q}_{\mathrm{r} 1}^{\mathrm{o}}=1, \tilde{q}_{i 2}^{\mathrm{o}}=0.25^{4}, t_{\mathrm{g} 1}=1, t_{\mathrm{k} 2}=0.25$. $H_{1}=H_{2}=1, N=0.1$. The cutoff frequency is given by $v_{\mathrm{c}} / c_{0} T_{\mathrm{gi}}=1 / 4000$.
Results using hybrid method for spectral calculation with one band optically thick

The two-flux solution computed by iteration had more rapid convergence when the diffusion method was used in the optically thick band rather than the two-flux method. Results from the hybrid method for a two-band calculation are in Fig. 8 for refractive indices of $n=1$ and 2 . The boundary temperatures are predicted quite well and the hybrid curves deviate only moderately from the exact solutions within the layer in the regions where the curvature is largest. Good predictions are obtained for the heat transfer $\tilde{q}_{101}$ through the layer.

\section{CONCLUSIONS}

The prediction of temperature distributions and heat transfer is carried out in semitransparent layers heated on both sides by radiation and convection so that the boundary temperatures are not specified and must be determined in the solution. The layers have refractive indices larger than one, and isotropic scattering is included. Three methods were developed for performing spectral calculations. The two-flux method was found to give excellent agreement with exact numerical solutions for all the conditions considered. The diffusion method was developed for spectral calculations in multilayered media and was found to give good predictions for large optical thicknesses. The diffusion results are much easer to evaluate than a numerical solution of the radiative transfer equations for a multilayered region with several spectral bands. A hybrid method was developed that can be applied when there are optically thin and optically thick spectral bands. This gave good temperature and heat flux predictions, and was easier to evaluate than the two-flux method when a spectral band is optically thick.

\section{REFERENCES}

1. R. Gardon, Calculation of temperature distributions in glass plates undergoing heat-treatment, J. Am. Ceramic Soc. 41, 200- 209 (1958).

2. C. M. Spuckler and R. Siegel, Refractive index effects on radiative behavior of a heated absorbing-emitting layer, J. Thermophys. Heal Transfer 6, 596-604 (1992).

3. C. M. Spuckler and R. Siegel, Refractive index and scattering effects on radiative behavior of a semitransparent layer, J. Thermophys. Heat Transfer 7, 302310 (1993).

4. C. M. Spuckler and R. Siegel, Refractive index and scattering effects on radiation in a semitransparent laminated layer, J. Thermophys. Heat Transfer (in press).

5. R. G. Sidall, Flux methods for the analysis of radiant heat transfer, Proceedings of the Fourth Symposium on Flames and Industry, Paper 16, pp. 169-179, The Institute of Fuel (1972).

6. R. Siegel and J. R. Howell, Thermal Radiation Heat Transfer (3rd Edn). Hemisphere. Washington, D.C. (1992).

7. F. Malpica, A. Campo and A. Tremante, Contribution of thermal radiation to the temperature profile of semi- 
transparent materials, High Temp-High Pressures 18, 35-41 (1986).

8. A. Tremante and F. Malpica, Contribution of thermal radiation to the temperature profile of ceramic composite materials, ASME Paper 93-GT-325, International Gas Turbine and Aeroengine Congress and Exposition. Cincinnati, Ohio, 24-27 May (1993).
9. J. C. Richmond, Relation of emittance to other optical properties, J. Res. Nat. Bureau Stand. 67C, 217-226 (1963).

10. J. R. Howell and M. E. Goldstein. Effective slip coefficients for coupled conduction-radiation problems. J. Heat Transfer 91, 165-166 (1969). 